\title{
Improving Access and Utilization of Maternal Healthcare Services through Focused Antenatal Care in Rural Ghana: A Qualitative Study
}

\author{
Umar Haruna $\mathbb{D}^{\mathrm{D}}$, Gordon Dandeebo, and Sylvester Z. Galaa \\ University for Development Studies, Department of Social, Political and Historical Studies, Wa Campus, Ghana \\ Correspondence should be addressed to Umar Haruna; humar@uds.edu.gh
}

Received 17 January 2019; Revised 1 June 2019; Accepted 17 June 2019; Published 1 July 2019

Academic Editor: Carol J. Burns

Copyright (c) 2019 Umar Haruna et al. This is an open access article distributed under the Creative Commons Attribution License, which permits unrestricted use, distribution, and reproduction in any medium, provided the original work is properly cited.

Improved access to and utilization of various maternal healthcare services have been seen as the panacea to poor maternal and child health outcomes characterizing many developing countries. Focused Antenatal Care (FANC) replaced the regular antenatal care model about a decade and a half ago. This study sought to document empirical outcomes of how the FANC approach translates access and utilization of maternal health services into positive maternal health outcomes. We utilized a descriptive qualitative design and analysis. We applied key informant interviewing to collect data from 206 respondents consisting of 140 women in their fertility age and 66 health workers across 14 communities in the study district. We found that FANC has been widely implemented across the district with most of the required services integrated into the existing healthcare delivery system. Overall, there has been successful implementation of FANC in the district, resulting in several benefits including the increased utilization of maternal healthcare services, acceptance of family planning, increased skilled delivery, and utilization of postnatal care (PNC) services. This notwithstanding, a number of issues need to be addressed to improve FANC services. These include provision of adequate infrastructure, essential supplies, communication and transportation systems, and manpower and adoption of positive sociocultural practices. No effort should be spared in providing these to sustain the successes and ensure sustainability of FANC.

\section{Introduction}

Maternal mortality and morbidity continue to be a significant problem in low-income countries, despite a worldwide focus on the need to improve maternal health. An estimated $99 \%$ of all maternal deaths occur in developing countries and more than half occur in sub-Saharan Africa (SSA) [1]. Developing countries continue to grapple with a huge burden of maternal and child mortalities where approximately 800 girls and women died as a result of pregnancy and childbirth-related complications in 2015 in SSA [2].

The situation calls for the adoption of drastic measures to stem the tide. Among the various strategies adopted to curb this menace are improvement in access and utilization of antenatal care services, increase in number of women who are attended to by skilled healthcare personnel during child birth, and provision of relevant postpartum or postnatal health services to both mothers and babies $[3,4]$.
Antenatal care (ANC), the first on the list of interventions, is broadly defined as encompassing pregnancy-related services provided between conception and the onset of labor with the aim of improving pregnancy outcomes and/or the health of the mother and/or child [5]. This care involves a series of assessments and appropriate treatments covering three components: monitoring of the health status of the woman and the fetus; provision of medical and psychosocial interventions; and support and health promotion [5].

ANC has over the years proven to be a good panacea to the problem of maternal and child mortalities. It is a key indicator of the Sustainable Development Goal (SDG) 3 , target 3.1, reducing the global maternal mortality ratio to less than 70 per 100,000. Among others, ANC is commonly understood to have beneficial impact on pregnancy and birth outcomes through early diagnosis and treatment of complications as well as promoting the health of the pregnant woman through nutrition [6]. ANC services create the opportunity 
for service providers to establish contact with the woman to identify and manage current and potential risks and problems during pregnancy [7]. It also creates the opportunity for the woman and her care providers to establish a delivery plan based on her needs, resources, and circumstances [8]. Again it creates opportunity for screening for such conditions as HIV and Sexually Transmitted Infections among others. The main impact of ANC is that it can lead to a reduction in severe anemia and cases of obstructed labor, and it improves the treatment of medical conditions [9]. Although ANC alone cannot prevent all obstetric emergencies, the information provided during ANC services can go a long way to ensure the successful management of pregnancies and the subsequent wellbeing of the child [10].

Historically, the traditional ANC service model was developed in the early 1900s. This model assumed that frequent visits and classifying pregnant women into low and high risk by predicting the complications ahead of time constituted the best way to care for the mother and fetus. The traditional approach was subsequently replaced by focus antenatal care, a goal oriented antenatal care approach, which was recommended and adopted by WHO in 2002 [11]. This was known as Focused Antenatal Care (FANC). The FANC departs from the usual facility based pregnancy classes concept of the previous ANC. FANC is client based and the site of its service is not limited to a health facility; this allows for the provision of tailored services at times and places most convenient to clients. It was believed that this approach would reduce barriers to ANC service utilization, improve access especially to rural women, and enhance quality of the services [12].

According to Kearns, Hurst, Caglia, and Langer [13], a multicountry randomized trial in 2001 assessed the effectiveness of a newer care model, Focused Antenatal Care, compared to the traditional ANC model. Overall, providers tolerated the new FANC model, women in both models of care were generally satisfied with services, and FANC cost the same or less than the traditional care model [14]. Focused or goal oriented ANC services provide specific evidence-based interventions for all women, carried out at certain critical times in the pregnancy [15].

FANC shifted the focus of care from the quantity to the quality of visits. Rather than identifying women as "high risk" or "low risk" and providing care accordingly, this model operates under the principle that all women are potentially at risk for developing complications and should receive individualized care and essential evidence-based interventions throughout their pregnancies [12]. Kearns and colleagues intimate that it is important to note that the basic FANC model is grounded in the idea that the majority of pregnancies do not involve complications [13]. Therefore, rather than using routine risk indicators such as maternal height to determine risk early on, the individualized, targeted approach of the FANC model aims to detect complications as they arise [16].

The principles of FANC for women are to provide advice, education, reassurance, and support; to address and treat the minor problems of pregnancy and; to provide effective screening during pregnancy [17]. The basic FANC model involves four antenatal visits that include individual counseling, targeted assessments, and the provision of safe, cost-effective, and evidence-based interventions [13]. Each of the four main visits consists of a well-defined set of activities related to three equally important general areas, namely, screening for conditions likely to increase adverse outcome, providing therapeutic interventions known to be beneficial, and educating pregnant women about planning for a safe delivery, emergencies during pregnancy, and how to deal with them [18]. The fourth visit of the pregnant woman to the facility requires the health service provider to review an individualized birth plan guide. With this guided plan, the healthcare provider discusses planned birth not exempting safe place of delivery and relevance of skilled birth attendant with the client and partner [19]. An underlying principle of FANC is the integration of care through health promotion, disease prevention, detection and treatment of existing diseases, and birth preparedness [20].

The FANC model recognizes that the antenatal period is a key entry point for many women into the health system, and so the model integrates ANC with care and counseling related to several other conditions. Women are immunized against tetanus and tested and treated for anemia as well as vitamin A or iodine deficiencies [12]. They also receive testing and treatment for HIV/AIDS, STIs, malaria, and tuberculosis [16]. In addition to a reduction in the possible visits from sixteen under the traditional model to four under FANC, the new model actively incorporates families and spouses into the process and encourages two-way communication through interactive counseling as opposed to the one-way health education of traditional ANC (Yengo, 2009), [12].

The FANC model recommends that visits should take place before 12 weeks, at 26 weeks, at 32 weeks, and between 36 and 38 weeks [8]. To account for the late enrolment in ANC observed in many developing countries, women who enroll late are expected to receive the care required for their specific gestational period as well as any preceding appointments. The WHO also recommends that formal systems are established to identify patients who have missed appointments and that mechanisms are in place to arrange follow-up visits [20]. In sum, FANC is intended to reduce waiting times, increase the time spent educating women about pregnancy-related issues, and stimulate the use of skilled assistance at birth $[7,16]$.

The potential of FANC is enormous, but its impact on the reduction of maternal deaths and its contribution to improved healthcare access and utilization remain contentious. Even though there is vast literature suggesting that FANC plays a positive role in improving maternal and child health outcomes in SSA, very little qualitative evidence exists in this regard. Studies by Nyarko et al. [15]; Yengo (2009); Kearns et al. [13]; Baffour-Awuah et al. [12]; Sumankuuro, Crockett, and Wang [21]; and Kuuire et al. [8] have all documented some benefits of FANC.

Research indicates that a good majority (96\%) of pregnant women in Ghana receive ANC from a trained provider, such as a doctor, nurse/midwife, or an auxiliary midwife [22]. But there are significant disparities between urban and rural communities [23]. The seemingly high utilization of ANC services however has not led to the expected commensurate 
reduction in maternal mortalities [24]. The Ghana $\mathrm{MoH}$ reports that inappropriate utilization of services or poor quality of services may account for this [24]. Furthermore, although almost all Ghanaian women seek antenatal care from a health professional and four in five women seek postnatal care, less than half of all women receive all three maternity care components (antenatal care, care at birth, and postnatal care) from a skilled provider (GSS, 2011).

The failure to properly utilize ANC services may also be attributed to other factors including how and where ANC services are provided [25, 26]. The issue of how and where ANC is provided brings into focus the nature of available evidence on FANC's implementation in Ghana and elsewhere. Although previous studies have established the value of FANC $[6,9,12,13]$, only a few make reference to the primary healthcare context in which FANC has operated. Dickson, Darteh, and Kumi-Kyereme [27] suggested that increased utilization and improved access to ANC could be attributed to the Community-Based Health Planning and Services (CHPS). Also, Naariyong et al. [28] made similar assertions when they indicated that 'pregnant women benefitted from better technical process quality of ANC services under the CHPS initiative than that found in non-CHPS areas in Ghana, expressly because of the design of the CHPS intervention'.

There is however a paucity of qualitative evidence on FANC implementation, especially within the context of existing primary healthcare system. For instance, how has implementation of FANC services harmonized alongside a local primary healthcare delivery system such as CHPS? In what way(s) has FANC contributed to improved access and utilization of complementary maternal health services such as skilled delivery, family planning, and postnatal care? What are the challenges confronting the implementation of FANC within local health landscape?

The paper discusses attempts made to translate access and utilization of maternal health services into positive maternal health outcomes based on the FANC approach using CHPS as the vessel. It examines the contributions of FANC to improved access and utilization of maternal healthcare services in rural communities in Ghana's Upper West Region. Understanding the context-specific benefits and challenges of differing populations in the study district is a necessary step for shaping future policies in order to impact positively on maternal and child health.

\section{Methodology}

2.1. Study Area. The study was carried out in the Daffiama, Bussie, Issa district of the Upper West Region of Ghana. The district was created in 2012, which makes it one of the new districts with barely three years in existence. It is bordered to the south by Wa municipal, west by Nadowli-Kaleo district, north by Sissala West district, and east by the Wa East district. It covers a total land area of $567.6 \mathrm{~km}^{2}$. The 2010 population census estimates that the district has a total population of 42,081 with 20,073 males and 22,008 females.

The district can be considered relatively deprived in terms of social, educational, and health infrastructure. In terms of health, the district has only five health centers, no hospital, and twelve functional Community-Based Health Planning and Service (CHPS) compounds [29]. The district has no resident medical doctor, one medical assistant, no functioning medical laboratory, and no emergency ambulance. The five health centers and 12 CHPS facilities are staffed by an estimated health worker population of about ninety.

The poor and socially marginalized populations in any given country tend to have the worst health status and access to professional health services. Rural populations, in particular, tend to suffer higher mortality rates than urban populations, and within urban populations, mortality is higher among the poor [8]. As such we found it imperative to examine how the FANC experiment in this deprived rural district contributed to maternal healthcare delivery outcomes, particularly in bridging the gap between attendance to antenatal visits and utilizing delivery and child health services.

2.2. Research Approach. A descriptive qualitative study design was used. This design allowed for an in-depth snapshot examination of the FANC program in the district. Using a purposive sampling method, we selected fourteen communities within the district. Target respondents were nurses and midwives working within the selected communities. The sample also included pregnant women and women with young children five years or younger. Finally we incorporated Traditional Birth Attendants (TBAs) into our sample as local auxiliary healthcare providers supporting mainstream healthcare delivery.

Pregnant women resident in the selected communities were usually selected with the assistance of nurses at the health facility when they attended ANC or by TBAs through their various pregnancy clubs (mother to mother support groups). This initial step was followed by a snowball sampling approach (thus, pregnant women/ANC attendees referred the researchers to other pregnant friends or relatives). Ultimately, the number of Women in Fertility Age (WIFA) respondents was determined by the point of saturation, whereby no further novel insights were identified from interviews [9]. For nurses and midwives, all available staff in the selected communities were included in the sample automatically. Once written permission was granted from the regional health directorate, all concerned nurses were contacted and their consent to participate was sought. TBAs were recruited with the help of personal contacts within the community.

Individual in-depth interviews and Focus Group Discussions (FGDs) were the main techniques used to collect data. Respondents provided views on the FANC, utilization of FANC, benefits of FANC, challenges of FANC, and maternal healthcare service delivery. Two FGDs, one for pregnant women and the other for nursing mothers, elicited information on the contributions of FANC to maternal healthcare services and the main challenges confronting its implementation. The research team conducted interviews in either Dagaare, Sissala, or English based on the preference of the respondent(s). With the respondents' consent, responses were recorded and later transcribed and translated into English for analysis. 
TABLE 1: Distribution of respondents by community and category.

\begin{tabular}{|c|c|c|c|c|c|c|c|}
\hline Community & EN Nurses & $\mathrm{CHO}$ & $\mathrm{CHN}$ & TBA & Midwives & Total & WIFA \\
\hline Challa & 1 & 1 & 1 & 1 & 0 & 4 & 8 \\
\hline Jinpensi & 0 & 1 & 1 & 1 & 0 & 2 & 9 \\
\hline Bussie & 3 & 0 & 2 & 1 & 1 & 7 & 12 \\
\hline Issa & 4 & 0 & 3 & 1 & 0 & 8 & 14 \\
\hline Kamehego & 0 & 1 & 0 & 1 & 0 & 2 & 6 \\
\hline Kojokperi & 1 & 1 & 1 & 1 & 0 & 4 & 11 \\
\hline Daffiama & 3 & 0 & 2 & 1 & 2 & 8 & 16 \\
\hline Fian & 2 & 0 & 2 & 1 & 1 & 6 & 10 \\
\hline Tabiesi & 1 & 1 & 1 & 1 & 0 & 4 & 11 \\
\hline Duang & 0 & 1 & 1 & 1 & 0 & 3 & 8 \\
\hline Owlo & 1 & 1 & 1 & 1 & 0 & 4 & 9 \\
\hline Touri & 1 & 1 & 1 & 1 & 0 & 4 & 11 \\
\hline Jolinyiri & 1 & 1 & 1 & 1 & 0 & 4 & 8 \\
\hline Wogu & 1 & 1 & 1 & 1 & 1 & 5 & 7 \\
\hline Total & 19 & 10 & 18 & 14 & 5 & 66 & 140 \\
\hline
\end{tabular}

Source: field data, 2015.

Individual interviews were commonly conducted in respondents' homes or, in the case of health workers, their place of work. Data collection and analysis were carried out in parallel, whereby emerging themes could be identified and incorporated into revised interview guides to provide a comprehensive insight into the relevant topics [9]. The dual techniques ensured the reliability of the findings.

The analysis of narrative data on similar topics from multiple sources allows for comparison of perspectives and triangulation [30]. Drawing on Sakeah et al. [30], analytical model, the narratives were first sorted by source (nurses, TBA, and WIFA), and then by instrument (FGD or interview) and multiple readings of the interviews and FGD data conducted. Topics discussed by respondents were noted, identifying those that were recurrent across interviews and discussions as well as those that appeared to be atypical or "outliers" in response to each question. In the second stage, the narratives were re-read, discussing larger themes that emerged across the respondents and across items. The narratives were supported by content analysis of existing documents such as annual health reports, which augmented the views and opinions of respondents.

\section{Results}

3.1. Demographic Characteristics of Respondents. Table 1 presents the distribution of respondents by category and community. In all, there were 66 health workers, made up of 14 TBA, 5 midwives, 18 community health nurses, 19 enrolled nurses, and 10 community health officers/registered staff nurses. TBAs included an average of 4 health workers per community, with the lowest number being 2 and the highest number being 8 health workers. The majority, 57 (86\%), of health workers were female; only $9(14 \%)$ were male.

Table 2 presents demographic features of the WIFA respondents. The age distribution shows a relatively young
TABLE 2: Demographic features of WIFA respondents.

\begin{tabular}{lcc}
\hline Variable & Frequency & $\%$ \\
\hline Age & & \\
\hline $15-19$ & 14 & 10 \\
$20-29$ & 52 & 37 \\
$30-39$ & 46 & 33 \\
$40-49$ & 28 & 20 \\
Total & 140 & 100 \\
\hline Reproductive Status & & \\
\hline Currently Pregnant & 84 & 60 \\
Not currently Pregnant & 56 & 40 \\
Total & 140 & 100 \\
\hline Birth Parity & & \\
\hline First Pregnancy & 37 & 26 \\
1-3 & 78 & 18 \\
$4-7$ & 25 & 100 \\
Total & 140 & \\
\hline
\end{tabular}

Source: field data, 2015.

sample with the vast majority (80\%) falling within the ages of 15 and 40 . Only $20 \%$ were aged over 40 years. Individually, however, the respondents within the ages of 20 and 29 were the highest, representing about $37 \%$ of this sample. This was followed by respondents within the ages of 30 and 39 , accounting for $33 \%$ of the sample. The remaining $10 \%$ were between the ages of 15 and 19, representing the least number of respondents.

The current reproductive status of the WIFA respondents as shown in Table 2 was divided into pregnant and nonpregnant women. The women who were currently pregnant represented $60 \%$ of this sample while the remaining $40 \%$ were not pregnant but were nursing children aged one month to four years. The birth parity of the 140 women in their fertility 
ages sampled indicted that first time mothers constituted $26 \%$ of that sample, while more than half $(56 \%)$ had gone through one to three childbirths. The remaining $18 \%$ had the highest birth parity of four to seven births. No respondent had more than seven births.

3.2. Contributions of FANC to Maternal Healthcare Services. One of the main issues examined by this study was how FANC was improving maternal healthcare service outcomes in the district. From the data collected, the FANC's approach is reported to be yielding positive results. The main areas where FANC has made critical contributions include overall ANC attendance, skilled delivery, increased PNC attendance, and improved reception of family planning education.

3.2.1. Antenatal Care. The implementation of FANC has brought about an improvement in the acceptability, access, and utilization of ANC services. The ANC registration and attendance in the district have seen a steady rise since the FANC approach was reemphasized. Available records indicate an annual increase of 29, 32, and 36 percent for the years 2012, 2013, and 2014, respectively [31]. This brought the ANC coverage to $88.7 \%$ in the year 2014, which represents a vast improvement over the 2013 coverage of $69.3 \%$ [32]. This was corroborated by health workers who reported,

I have been working in this district for the
past seven years and can attest that pregnant
women now utilize antenatal care more than
ever. I think the new model together with
the CHPS initiative has actually revolution-
ized ANC. More and more women now partic-
ipate. (32 year old Midwife, Key Informant
Interview)

Another health worker said,

Indeed there has been a marked improvement in $A N C$, there has also been an increased awareness and cooperation between expectant mothers and health workers. Definitely the utilization numbers have increased. (28 year old Community Health Nurse, Key informant Interview)

When asked why there was this steady increase of ANC utilization, respondents attributed it to the flexible nature of FANC, increased health worker collaboration with local health structures (TBAs, mother to mother support groups) through the CHPS initiative. The following responses best exemplifies these assertions:

In my opinion we have more women attending ANC services because of the flexibility of the system. We now encourage walk in ANC services. In the past we used to undertake ANC as if it was a clinic session. We had specific days where we undertook pregnancy classes, counseling and screening for danger signs all on specified days. It used to be quite cumbersome then, long queues and space constraints hampered efficient delivery of service. (38 year old Senior Midwife)
Another discussant shared her opinion thus:

When I was pregnant with my second child, ANC used to be my greatest worry. There used to long queues at the facility. In fact everything involved long queues and waiting periods; for instance, you had to 'do a line' for weighing, another for vital signs screening, another to collect medicine and so forth. It was really tiresome. So after a few visits I became reluctant going for ANC checkups. (40 year old woman, FGD)

A male $\mathrm{CHO}$ stated,

The walk in ANC has really helped us a lot in doing one on one counseling. It also allows us to be thorough since you usually have one client to attend to at a time. We can now see clients anytime they come in or even in their homes. (26 year old $\mathrm{CHO}$, Key Informant)

Another discussant observed,

Right from registration for ANC through to PNC, members of my community's mother to mother support group have been instrumental. They hounded me to register, reminded me of the need to regularly check up and saw to it that the $\mathrm{CHO}$ visited me consistently until I put to bed. (35 year old woman, FGD)

3.2.2. Skilled Delivery. Another positive outcome of the FANC is the improvement of skilled delivery. It was found that there had been improvements in the utilization of health facilities for delivery of babies. With respect to our sample, out of the 56 women who had given birth within the last three years prior to the study, $49(87.5 \%)$ delivered with a skilled personnel. This is much higher than the district average of $53.7 \%$ reported in the Ghana Health Service's Annual Regional Report for 2015. Skilled delivery increased steadily from $58.2 \%(17,285)$ in 2013 to $63.4 \%(19,204)$ in 2014 and dropped slightly to $61.9 \%(19,123)$ in 2015 . The region achieved an approximate $62 \%$ coverage, exceeding the annual target of $60 \%[32]$.

Home deliveries have been virtually outlawed in most communities, with offenders required to pay fines for flouting that rule. With the help of the CHPS program, commitment by the elders and people of the community, health volunteers, TBAs, and mother to mother support groups, pregnant women have been sensitized to deliver their babies at appropriate health facilities. The reasons for this improvement are highlighted in the following responses from a health worker, a nursing mother, and a community health volunteer:

We are very happy with the way women now strive to deliver with a skilled health worker. I can say it is because of the numerous health campaigns we have undertaken over the years. It is also because of the way we treat them during ANC, for instance we do defaulter tracing and home visits to ensure that all women who should be undertaking 
ANC do so. Similarly, we monitor and recommend women who are almost due to the nearest health facilities to prepare for deliver. No home delivery is allowed. This certainly has improved the rate at which women undertake supervised delivery. (27 year old $\mathrm{CHO}$, Key Informant)

A women who recently gave birth added,

These days it will be difficult to give birth at home. If you go through all the antenatal sessions, you will definitely be very prepared for delivery. In my case, my midwife visited me severally to conduct antenatal even in my house. When it was three weeks left for me to put to bed, I was asked to go to Bussie which has a health center to prepare for delivery. In Bussie I stayed with my husband's relatives and visited the clinic every day until I was almost due. I delivered a bouncing baby girl ten days after my referral. I was only admitted just 24 hours prior to delivery. (34 year old Nursing mother)

Another respondent, a community health volunteer, argue,

Skilled delivery has improved because of the birth preparedness approach. Again the concerted efforts of the midwife, TBA and the women's group in this community also played a crucial role this improvement. I will also attribute this success to the elders of the community who have instituted a fine to all those who deliver at home. I will not leave out the efforts of my colleague volunteers who see to it that all women attend antenatal and when they are ready we ensure that they seek supervised delivery. (40 year old Health Volunteer)

3.2.3. Expansion in Postnatal Care. Another revelation of this study is that the utilization of PNC services had seen an improvement as well. As a direct consequence of delivering at health facilities under the supervision and care of health workers, nursing mothers were encouraged to make use of PNC services. It was revealed that many women who delivered at the facilities did make their first PNC visit within the first week after delivery as recommended. For instance, $96.4 \%$ of nursing mothers (54 out of 56) in our sample had utilized PNC services within 48 hours after delivery. Similarly, the district in 2015 attained 99.1\% (791/798) [32].

Additionally, women who had some complication after delivery were able to seek additional care from that facility or got referred to better equipped facilities. From the discussions, it was discovered that both health workers and clients were particularly satisfied with the PNC services available to them. They attributed the improvement in attitudes toward utilizing PNC to the elaborate education and counseling they received during ANC. A respondent explained,

In the past we did not know it was necessary to keep visiting the clinic even after delivery. But during ANC, we were taught that both mothers and children are vulnerable in the first weeks after delivery hence we should continue to see health professional to see to it that we are given the best treatment to ensure that both mother and child is safe. I think they have a point. At PNC several things were done including seeing to it that my wounds were healing properly, that my child weighed in, some immunizations were given and others planned. Breastfeeding issues were also discussed. (21 year old Nursing Mother)

3.2.4. Family Planning Services. Another improvement the FANC model is making is in the area of family planning. Through the health education and promotion component of FANC, pregnant women are counseled on several issues including how to space their births through the use of contraceptives. During ANC, the nurses take the opportunity to advise couples on the importance of family planning as well as the reproductive health dangers associated with frequent child birth. Nearly all women (99.4\%) in the sample indicted they had received some form of education on family planning and contraceptive use from health workers. Interestingly, 63 out of $140(45 \%)$ women in our WIFA sample admitted never using a contraceptive before their last childbirth. Although contraceptives coverage for the region has improved from $51 \%(91,779)$ in 2014 to $53 \%(98,352)$ in 2015 , the district recorded $44.2 \%$ in 2015 , an increase of about nearly $4 \%$ from the 2014 figure of $40.3 \%$ [32]. Contraceptives such as Depo, Implanon, pills, Norigynon, Jadelle, and condoms were reported to be the preferred methods over the years.

Responding to the link between FANC and family planning, respondents had the following to say:

As a health worker, one of our duties is to see to it that our clients are healthy and happy. As such we use every means possible to offer them advice on how to remain healthy. Family planning campaigns have been met with some resistance in the past. However, under the guise of the new FANC, which allow as more one on one time with the clients, we usually offer family planning advice as part of our routine ANC package. The advantage is that sometimes some clients receive this advice in the presence of their spouses and this proves to be a good way of attaining compliance. (23 year old $\mathrm{CHO}$, Key Informant)

Another respondent remarked,

Indeed I received a lot of family planning information during my ANC. The nurses convinced me and my husband to try and space out my subsequent pregnancies. They also introduced and explained to us how to use natural and artificial contraception to achieve these. The way they put the message, we were easily convinced and since then my husband and I have been more receptive to family planning messages 
in general and contraceptive use in particular. (32 year old Key informant)

\subsection{What Remains to Get FANC Working as Expected}

3.3.1. Infrastructure. As with many healthcare interventions, the respondents reported several challenges confronting FANC services provision. The challenges ranged from inadequate infrastructure, lack of adequate human resources, and logistical constraints to sociocultural practices. A major challenge confronting the implementation of FANC was attributed to the nature of healthcare infrastructure across the district. The district had five health centers and twelve functioning CHPS compounds. The lack of health facilities like a well-equipped laboratory was a major impediment for healthcare delivery and ANC services in particular. A critical component of the FANC is to conduct screening and laboratory investigations for pregnant women; the lack of a functioning diagnostic laboratory facility in the district posed a challenge to the implementation of FANC. As opined by a respondent,

\section{The lack of an equipped Lab in this district hampers our efforts to improve maternal and child health in this district. We always refer clients to Nadowli or Wa to undertake labo- ratory investigations. Due to the distance and sometimes cost involved, most often, the clients refuse to go or it takes too much time to get a results back. (A 29 year old, Registered Staff Nurse, Key Informant)}

3.3.2. Logistics, Equipment, and Essential Supplies. Associated with the inadequacy and the lack of essential infrastructure, the provision of FANC services is also hampered by lack of logistics, equipment, and essential supplies. It emerged that basic medical supplies for ANCs are usually in short supply. Supplies and equipment are usually inadequate or sometimes in short supply (syringes, gloves, gauze swabs, drugs, etc.). below:

A response that encapsulates this challenge is presented

It is incredible how the lack of logistics and basic equipment continue to be the bane of our efforts to implement FANC in this district. We always seems to have the shortage of one drug or another. We never have the full complement of the essential supplies that we need to carry out our duties. Our motorbikes for home visits are inadequate or lack fuel and maintenance. When there is shortage of supplies we ration, which means clients don't get the full complements of what they need. This erodes some of the gains we are making. (25 year old Enrolled Nurse, Key Informant)

3.3.3. Human Resources. Another startling revelation was that the lack of personnel at the various healthcare facilities and CHPS compounds is one of the most enduring constraints that affects FANC in the district. The respondents were unanimous in bemoaning the shortage of healthcare workers in their communities. The shortage of midwives to provide various services to clients was particularly profound to FANC. For instance, there are only five midwives present in the entire district. The regional distribution shows 1 midwife to 1,220 women, falling short of the WHO requirement of 1 midwife to 650 women [29].

It was obvious that FANC services were more attractive and user friendly; hence patronage had gone up over the years. The increasing number of clients and expanded services has not resulted in a commensurate increase in the number of health personnel, hence the understaffing. The issue of shortage of personnel is further highlighted in the following statements by a health worker and a client:

I can say that one of our major problems here is that we don't have the right number of staff working in this district, right from doctors, physician assistants, nurses and midwives, we are short of personnel. This affects our work immensely. We are usually overworked and we are sometimes too exhausted to give off our best. (35 year old Health Worker)

I can say that the number of nurses and midwives is woefully inadequate. Imagine only two nurses taking care of this large community. Sometimes they get overwhelmed when two or more women go into labor at the same time. In addition to lack of space and equipment, there are not enough midwives to help in deliveries. This also affects the number of minutes spent at the counseling sessions. (36 year old woman)

3.3.4. Telecommunication, Road, and Transport Services. Another major hindrance to FANC services was the bad nature of telecommunication services coupled with the poor nature of roads and the lack of proper transport systems. The quality of telecommunications network in the districts was found to be very poor. Making and receiving phone calls constituted a major difficulty across the district. With the exception of a few towns, many communities did not have good cellphone reception. During FGDs, respondents consistently asserted that there was rampant disruption of cellphone reception on regular basis.

Another major finding was the poor and inaccessible nature of roads in the district. As part of the home visit and defaulter tracing components of CHPS, health workers are required to make regular routine household visits to their clients to provide services including FANC to residents. However due to the deplorable nature of the roads as well as the inaccessible nature of some of the communities, this aspect of the FANC is regularly omitted by some health workers.

3.3.5. Constraining Cultural Practices. The interviews also unearthed evidence that FANC was hampered by some 
negative cultural practices. Some respondents reported that the late registration and attendance of ANC were a result of the traditional view that women should not hasten to reveal their pregnancy status. This was to 'prevent exposing the fetus and the mother to evil spirits'. This unhealthy prohibitions prevented women from even taking steps to confirm their pregnancy status, hence causing a delay seeking ANC services.

Another cultural issue that emerged from the discussion was gender inequalities between men and women. It was further reinforced that rural women had very little to say about their own reproductive health issues. Women lacked the autonomy to take many decisions relating to their reproductive health; hence they had to seek permission from their spouses or male relatives before embarking on any activity including seeking antenatal care. For fear of reprisal, some women most often refused to take up ANC until it was usually too late to offer essential FANC services.

Cultural dogmatism appeared to be another significant bottleneck hampering FANC in the district. Some respondents lamented that in spite of proactive measures by health workers and community leaders to ensure that all women utilized ANC services when pregnant and skilled delivery when giving birth, some isolated groups of people still resisted these laws, preferring instead to stick to outdated traditional medical practices.

A respondent reported,

The migrant Fulani ethnic group in this and nearby communities keep flouting our bye laws on home delivery. They prefer to deliver at home or in the 'bush', using their own traditional methods rather than visiting the health facility. Last year a pregnant Fulani woman died while trying to give birth at home. The members of the community were very furious, he husband had refused to allow her to attend ANC services and when she was due he forced her to deliver at home. We don't understand why they are so difficult to convince. (23 year old Nursing Mother)

\section{Discussion}

The study sought to highlight how FANC has been implemented under the auspices of CHPS in a rural Ghanaian district and how this contributed to improved health outcomes through improved utilization and access. The findings demonstrate that FANC has been implemented comprehensively in the Daffiama, Bussie, and Issa district on the platform of the CHPS program. The various findings offer novel insights into the role of the CHPS program as a vessel through which the delivery of a proven intervention to improve maternal and child healthcare has been successfully channeled. Overall, these findings enhance the existing evidence on leveraging community level programs to improve the acceptability and access to health interventions in deprived, resource constrained communities.

Our findings show that there has been a steady increase in the utilization of ANC services which is attributable to the flexible nature of FANC within the CHPS framework. FANC care is largely provided on personal basis, birth preparedness has been improved, and early detection of complications and provision for their management or treatment is now readily available. A particularly novel aspect of FANC under the CHPS is how clients are identified and registered. Women who are suspected to be pregnant are quickly identified by community members (in some cases designated mother to mother support group leaders or TBAs) within their community and presented to the health workers for confirmation. This ensures that women, confirmed to be pregnant, utilize ANC services early and are able to make the minimum of four visits recommended by the WHO. This confirms the assertions that community level structures if identified and harnessed appropriately can be useful tools in improving maternal health in rural areas [33-35].

The CHPS program, which permits one on one FANC services, has provided an impetus for increased utilization of services. FANC departed from the old pregnancy classes and clinic sessions module to an approach where ANC services are personalized to suit each client, usually one client at a time, involving ' a walk in anytime' basis and extended periods of counseling. FANC services has therefore been seamlessly synergized with the CHPS approach of home based visits and defaulter tracing. The key innovation of FANC to shift attention from risk categorization to focused care and maternal health at large has proved to be a master stroke toward the improvement of maternal health. The household visits component of CHPS, incorporated into FANC implementation, ensures a systematic and significant improvement in access, with clients being reached at their homes instead of having to make trips sometimes exceeding 10 kilometers to access care [36].

The implication of all these is that FANC, implemented within the CHPS framework, has reduced barriers to access and other impediments in terms of time constraints, improved privacy, and gave more time for counseling, timely testing, and treatment of pregnancy-related complications. We discovered that women who disliked the old format of ANC have a liking for the FANC version and this has greatly resulted in improved access and utilization.

The results have demonstrated that the expansion of services to include spouses and other immediate family members has also contributed significantly to the success of FANC implementation. More specifically, because counseling and education involve both husband and wife, there is a growing involvement of men in reproductive health issues. This has had a direct positive effect on health service utilization and increasing democratization of reproductive health decisions [37]. By motivating caretakers and spouses to accompany their pregnant wives to antenatal care facilities during service delivery, the FANC has gone a long way to stimulate interest in and understanding of the importance of antenatal care delivery as well as reinforcing maternal and child health support systems at the household level, thus resulting in the high patronage and utilization of care in the care. The FANC has also leveraged traditional community leadership to outlaw some obnoxious traditional maternal and health practices in the area. 
Similar to documented assertions by Villar \& Bergsjø, [38]; Babalola \& Fatusi, [7]; Pell et al. [9]; Dibaba et al. [6]; Kearns et al. [13], and Baffour-Awuah et al. [12], our findings also show that FANC has not only contributed to improving access and utilization of ANC, but has also brought about an increase in skilled delivery and improvement in attitudes toward PNC service utilization [30].

Like many interventions in the health sector, the FANC model under the CHPS is bedeviled with many challenges. These challenges are largely structural and include inadequate infrastructure, (regular) shortage of essential supplies, poor communication and transportation systems, lack of manpower, and negative sociocultural practices. These challenges collectively lower the quality of antennal and postnatal care delivered under the FANC as corroborated by other studies [13, 30, 39-41]. When medical supplies are in short supply, the direct consequence is the inability to treat clients and in some cases clients' ailments are worsened due to reduced medications. These essential supplies and equipment play a critical role in ensuring that FANC activities are carried out timeously and efficiently. These inadequacies limit the potential positive outcomes of FANC.

The lack of infrastructure, especially laboratory facilities, also makes it difficult to diagnose pregnancy complications and other risks timeously. The inability to do so hampered FANC services. Space constraints at CHPS compounds contribute to regular overcrowding, and delays in providing services regularly occurred. This has led to clients being frustrated and complaining that the situation resembled the old ways of ANC, where overcrowding was persistent [42]. Coupled with the above is the lack of the requisite number of health staff at the CHPS compound and other supporting health facilities.

Poor telecommunication and transportation systems in the district also have the potential to render the provision of FANC services ineffective. Poor roads, inaccessible communities, and broken down motor bikes as well as poor cellphone reception were serious obstacles to health service provision and FANC services in some of the communities covered. The net result of this was that referral of cases was sometimes done without the knowledge of receptor facilities. It also made it difficult for relevant personnel to be contacted in times of emergencies. In some instances where health workers needed to conduct some counseling or education on phone, this becomes impossible to do with the poor quality of cellphone reception in the area. Poor accessibility and lack of transportation also hampered effective home visits and defaulter tracing system by the CHPS. This confirms the findings of Mills et al. [39].

Finally, isolated negative sociocultural practices still posed a major problem in the utilization of FANC and other CHPS services (Dako-Gyekye et al., 2015). In spite of that, there have been strides by local communities to ensure that people adhere to the advice of health professional by utilizing healthcare services provided by nurses and midwives at CHPS compounds and health centers. Some communities have established by-laws to make this effective. Some pregnancy myths and taboos highlighted by previous studies [7, 33] are being countered, hence a reduction in late utilization of ANC.

\section{Conclusion}

FANC services delivered under the auspices of CHPS have been largely accepted and recognized as a very useful intervention in the improvement of access and utilization of maternal health services. The findings established that FANC has been widely and successfully implemented in a deprived and resource constrained district under the CHPS framework. FANC successful implementation comes with several benefits to the community, such as the steady improvement in maternal mortality and reduced child mortalities as a result of increased utilization of FANC, gradual acceptance of the family planning, increased skilled delivery, and utilization of PNC services.

However, the benefits accruing from FANC implementation could be easily eroded if certain challenges are not immediately addressed. Inadequate infrastructure, regular shortage of essential supplies, poor communication and transportation systems, lack of manpower, and negative sociocultural practices have been identified as the key challenges to health service provision and also impacted on FANC service provision.

The CHPS initiative has been found to be particularly useful in the implementation of the FANC; as such, no effort should be spared to ensure its sustainability and scalability to other areas of the country. Preventive and promotional health education campaigns have been particularly useful in changing attitudes toward ANC and other maternal services utilization; hence this must be encouraged and sustained. Community leaders should continue to promote collaboration between traditional health providers and the modern health professionals to deliver essential maternal and child health services. By-laws aimed at encouraging these collaborations must be strictly enforced to make them more effective.

Additionally, we recommend that steps should be taken by the Government, through the Ministry of Health and the Ghana Health Service, and other well-meaning individuals and groups to, in the medium to long term, assist the district with healthcare infrastructure including, but not limited to, hospitals, clinics, health centers, laboratories, and hospital equipment. However, in the short term, immediate assistance should be extended to these facilities to keep them functioning efficiently and effectively.

The human resource situation must also be addressed. Increasing the number of health workers and providers may also help reduce the current strain on the few CHPS health workers, which has diverted time away from essential components of FANC such as counseling and integrated services. This can be done by training more health professionals, particularly midwives, to reduce understaffing and to improve the delivery of CHPS services including FANC. It is imperative to ensure that the necessary supplies are available to provide the recommended interventions for each visit. The Ghana Health Service and other agencies responsible 
must ensure that the basic and essential supplies are always available in their right quantities and arrive on time to prevent shortages and rationing of these supplies.

\section{Data Availability}

The qualitative data used to support the findings of this study are included within the article.

\section{Ethical Approval}

The study adhered to all ethical concerns, by following all ethical guidelines for research laid down by the Ministry of Health, Ghana Health Service, and the University for Development Studies. Ethical approval was granted by the University for Development Studies Ethical Review Committee. The Ghana Health Service Regional Directorate also granted permission to visit the sampled health institutions and to have interactions with staff manning these institutions as part of the data collection process. The ethics guaranteed anonymity and confidentiality for all participants.

\section{Consent}

Verbal informed consent for audio recording of interviews and discussion sessions was sought from all participants of the study before the commencement of data collection.

\section{Conflicts of Interest}

The authors declare that they have no conflicts of interest.

\section{Acknowledgments}

We would like to extend our appreciation to the director and staff of the Regional Health Directorate of the Upper West Region, especially the CHPS Unit. We are also particularly grateful to the directors and staff of the Daffiama, Bussie, and Issa District Health Directorate for their invaluable assistance during the conduct of this study. We also owe a debt of gratitude to community leaders, health workers, and the respondents for their participation in the study.

\section{References}

[1] World Health Organization [WHO], "Maternal Mortality Factsheet," 2018, http:/www.who.int/news-room/fact-sheets/ detail/maternal-mortality.

[2] J. Sumankuuro, J. Crockett, and S. Wang, “The use of antenatal care in two rural districts of Upper West Region, Ghana," PLoS ONE, vol. 12, no. 9, p. e0185537, 2017.

[3] O. M. Campbell and W. J. Graham, "Strategies for reducing maternal mortality: getting on with what works," The Lancet, vol. 368, no. 9543, pp. 1284-1299, 2006.

[4] Z. S. Lassi, T. Mansoor, R. A. Salam, J. K. Das, and Z. A. Bhutta, "Essential pre-pregnancy and pregnancy interventions for improved maternal, newborn and child health," Reproductive Health, vol. 11, article no. S2, 2014.
[5] J. Hollowell, L. Oakley, J. J. Kurinczuk, P. Brocklehurst, and R. Gray, "The effectiveness of antenatal care programmes to reduce infant mortality and preterm birth in socially disadvantaged and vulnerable women in high-income countries: a systematic review," BMC Pregnancy and Childbirth, vol. 11, no. 1, article no. 13, 2011.

[6] Y. Dibaba, M. Fantahun, and M. J. Hindin, "The effects of pregnancy intention on the use of antenatal care services: systematic review and meta-analysis," Reproductive Health, vol. 10, no. 1, article 50, 2013.

[7] S. Babalola and A. Fatusi, "Determinants of use of maternal health services in Nigeria-looking beyond individual and household factors," BMC Pregnancy and Childbirth, vol. 9, no. 1, article no. 43, 2009.

[8] V. Z. Kuuire, J. Kangmennaang, K. N. Atuoye et al., "Timing and utilisation of antenatal care service in Nigeria and Malawi," Global Public Health, vol. 12, no. 6, pp. 711-727, 2017.

[9] C. Pell, A. Meñaca, F. Were et al., "Factors affecting antenatal care attendance: results from qualitative studies in Ghana, Kenya and Malawi," PLoS ONE, vol. 8, no. 1, Article ID e53747, 2013.

[10] C. L. Abou-Zahr and T. M. Wardlaw, Antenatal Care in Developing Countries: Promises, Achievements and Missed Opportunities: An Analysis of Trends, Levels and Differentials, World Health Organization, Geneva, Switzerland, 2003.

[11] S. Di Mario, V. Basevi, G. Gori, and D. Spettoli, "What is the effectiveness of antenatal care? (Supplement)," WHO Regional Office for Europe (Health Evidence Network), Copenhagen, 2005.

[12] A. Baffour-Awuah, P. P. Mwini-Nyaledzigbor, and S. Richter, "Enhancing focused antenatal care in Ghana: an exploration into perceptions of practicing midwives," International Journal of Africa Nursing Sciences, vol. 2, pp. 59-64, 2015.

[13] A. Kearns, T. Hurst, J. Caglia, and A. Langer, Focused antenatal care in Tanzania: delivering individualised, targeted, high quality care, Woman and Health Initiative: Maternal Health Task Force, 2014.

[14] J. Villar, H. Ba'aqeel, G. Piaggio et al., "WHO antenatal care randomised trial for the evaluation of a new model of routine antenatal care," The Lancet, vol. 357, no. 9268, pp. 1551-1564, 2001.

[15] P. Nyarko, H. Birungi, M. Armar-Klemesu et al., "Acceptability and feasibility of introducing the WHO focused antenatal care package in Ghana," FRONTIERS Final Report, Population Council, Washington, DC, USA, 2006.

[16] S. Gabrysch and O. M. R. Campbell, "Still too far to walk: literature review of the determinants of delivery service use," BMC Pregnancy and Childbirth, vol. 9, no. 1, p. 34, 2009.

[17] A. C. G. Breeze and L. H. Kean, "Routine antenatal management at the booking clinic," Obstetrics, Gynaecology and Reproductive Medicine, vol. 20, no. 1, pp. 1-6, 2010.

[18] M. A. Al-Ateeq and A. A. Al-Rusaiess, "Health education during antenatal care: the need for more," International Journal of Women's Health, vol. 7, pp. 239-242, 2015.

[19] World Health Organization [WHO], The world health report. making every woman and child count: policy brief one: integrating maternal newborn and child health programmes, WHO, Geneva, Switzerland, 2012.

[20] J. Villar and P. Bergsjo, WHO Antenatal Care Randomized Trial: Manual for the Implementation of the New Model, WHO, Geneva, Switzerland, 2002. 
[21] J. Sumankuuro, J. Crockett, and S. Wang, "Antenatal care on the agenda of the post-millennium development goals in northern Ghana," International Journal of Innovation and Applied Studies, vol. 18, pp. 341-352, 2016.

[22] P. Dako-Gyeke, M. Aikins, R. Aryeetey, L. Mccough, and P. B. Adongo, "The influence of socio-cultural interpretations of pregnancy threats on health-seeking behavior among pregnant women in urban Accra, Ghana," BMC Pregnancy and Childbirth, vol. 13, no. 1, artilce no. 211, 2013.

[23] Ghana Demographic and Health Survey (GDHS), Report on Maternal Health, GSS, GHS, and ICF Macro, Accra, Ghana, 2014.

[24] Ministry of Health [MoH], Holistic Assessment of the Health Sector Program of Work 2013, Accra, Ghana, 2014.

[25] B. Simkhada, E. R. Van Teijlingen, M. Porter, and P. Simkhada, "Factors affecting the utilization of antenatal care in developing countries: systematic review of the literature," Journal of Advanced Nursing, vol. 61, no. 3, pp. 244-260, 2008.

[26] M. A. Magadi, N. J. Madise, and R. N. Rodrigues, "Frequency and timing of antenatal care in Kenya: explaining the variations between women of different communities," Social Science \& Medicine, vol. 51, no. 4, pp. 551-561, 2000.

[27] K. S. Dickson, E. K. M. Darteh, A. Kumi-Kyereme, and B. O. Ahinkorah, "Determinants of choice of skilled antenatal care service providers in Ghana: analysis of demographic and health survey," Maternal Health, Neonatology and Perinatology, vol. 4, no. 1, artilce no. 14, 2018.

[28] S. Naariyong, K. C. Poudel, M. Rahman, J. Yasuoka, K. Otsuka, and M. Jimba, "Quality of antenatal care services in the birim north district of ghana: contribution of the community-based health planning and services program," Maternal and Child Health Journal, vol. 16, no. 8, pp. 1709-1717, 2012.

[29] Ghana Health Service [GHS], Annual Health Sector Report, 2013, Ghana Health Service, Accra, Ghana, 2014.

[30] E. Sakeah, L. McCloskey, J. Bernstein, K. Yeboah-Antwi, S. Mills, and H. V. Doctor, "Is there any role for community involvement in the community-based health planning and services skilled delivery program in rural Ghana?" BMC Health Services Research, vol. 14, no. 1, article no. 340, 2014.

[31] Ghana Health Service [GHS], Annual Upper West CHPS Report, 2015, Ghana Health Service, Accra, Ghana, 2015.

[32] Ghana Health Service [GHS], Upper West Annual Regional Health Report, 2015, Ghana Health Service, Accra, Ghana, 2016.

[33] A. Sato, "Does socio-economic status explain use of modern and traditional health care services?" Social Science \& Medicine, vol. 75, no. 8, pp. 1450-1459, 2012.

[34] U. Haruna, M. M. Kansanga, and S. Galaa, "Examining the unresolved conundrum of Traditional Birth Attendants' involvement in maternal and child health care delivery in Ghana," Health Care for Women International, pp. 1-19, 2018.

[35] H. Woods, U. Haruna, I. Konkor, and I. Luginaah, "The influence of the community-based health planning and services (CHPS) program on community health sustainability in the Upper West Region of Ghana," International Journal of Health Planning and Management, vol. 34, no. 1, pp. e802-e816, 2018.

[36] F. A. Johnson, F. Frempong-Ainguah, Z. Matthews et al., "Evaluating the impact of the community-based health planning and services initiative on uptake of skilled birth care in Ghana," PLoS ONE, vol. 10, no. 3, Article ID e0120556, 2015.

[37] S. Lewis, A. Lee, and P. Simkhada, "The role of husbands in maternal health and safe childbirth in rural Nepal: a qualitative study," BMC Pregnancy and Childbirth, vol. 15, no. 162, 2015.
[38] J. Villar and P. Bergsjø, "Scientific basis for the content of routine antenatal care I. Philosophy, recent studies, and power to eliminate or alleviate adverse maternal outcomes," Acta Obstetricia et Gynecologica Scandinavica, vol. 76, no. 1, pp. 1-14, 1997.

[39] S. Mills, E. Bos, E. Lule, G. N. V. Ramana, and R. Bulatao, "Obstetric care in poor settings in Ghana, India, and Kenya," HNP Discussion Paper Series, 2007.

[40] A. W. Al Serouri, A. Al Rabee, M. Bin Afif, and A. Al Rukeimi, "Reducing maternal mortality in Yemen: challenges and lessons learned from baseline assessment," International Journal of Gynecology and Obstetrics, vol. 105, no. 1, pp. 86-91, 2009.

[41] K. N. Atuoye, J. Dixon, A. Rishworth, S. Z. Galaa, S. A. Boamah, and I. Luginaah, "Can she make it? transportation barriers to accessing maternal and child health care services in rural Ghana," BMC Health Services Research, vol. 15, no. 1, article no. 333, 2015.

[42] E. Arthur, "Wealth and antenatal care use: implications for maternal health care utilisation in Ghana," Health Economics Review, vol. 2, no. 1, artilce no. 14, pp. 1-8, 2012. 


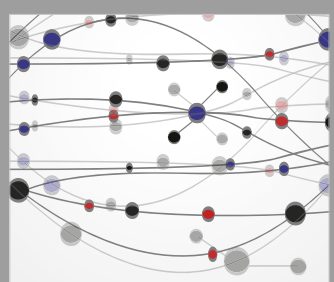

The Scientific World Journal
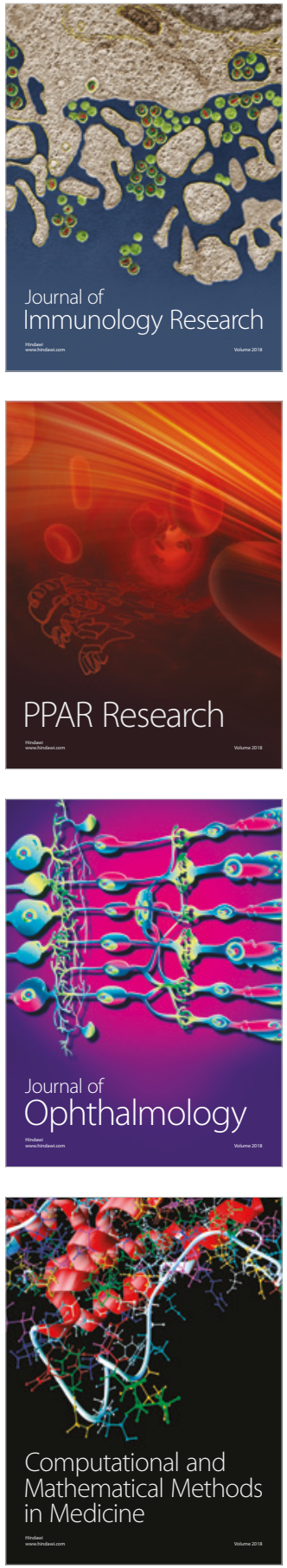

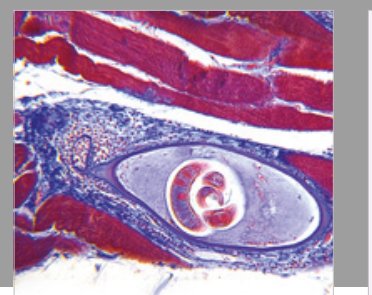

Gastroenterology Research and Practice

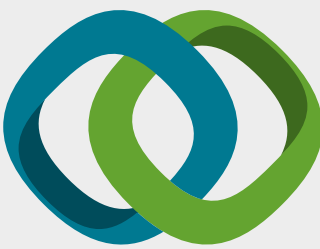

\section{Hindawi}

Submit your manuscripts at

www.hindawi.com
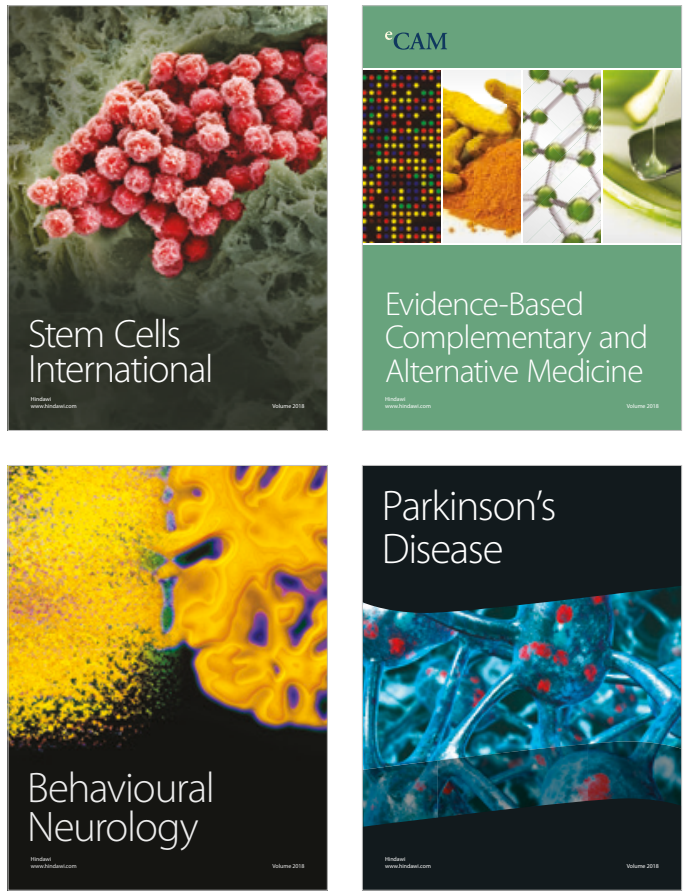

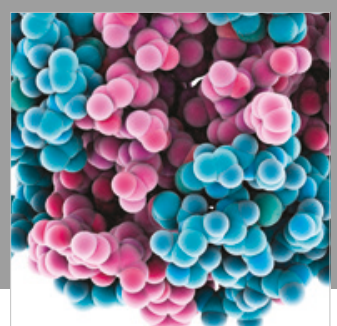

ournal of

Diabetes Research

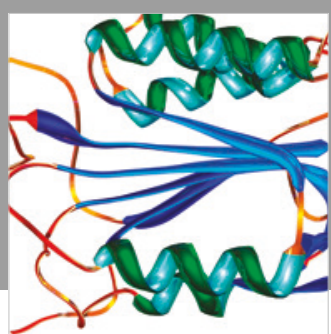

Disease Markers
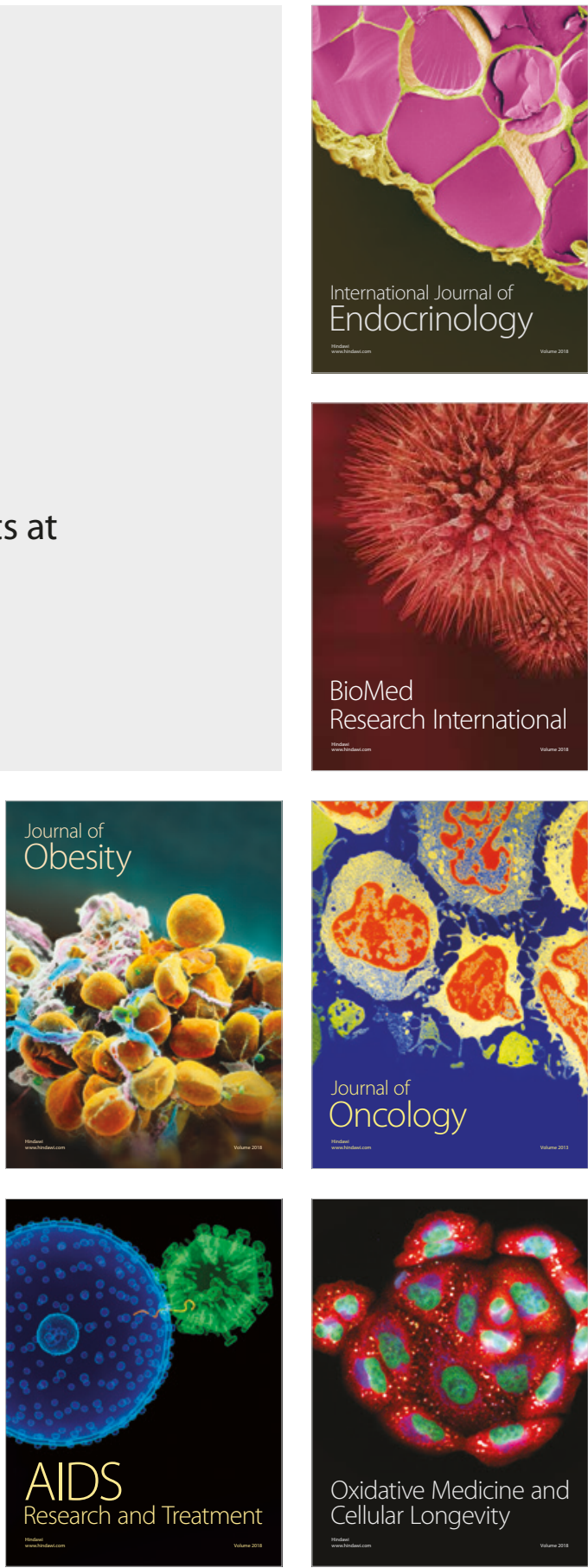